Military Technical College

Kobry El-Kobbah, Cairo, Egypt

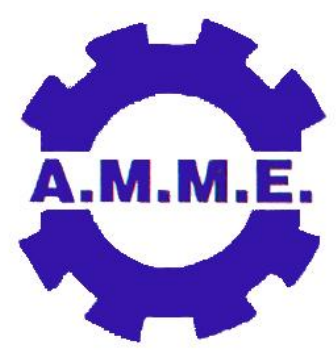

$14^{\text {th }}$ International Conference on Applied Mechanics and Mechanical Engineering.

\title{
MULTI-AGENT SYSTEM PATH-PLANNING USING AGENT PERCEPTUAL MODEL AND DYNAMIC ARTIFICIAL POTENTIAL FIELDS
}

\author{
M. H. MABROUK
}

\begin{abstract}
Artificial teams of mobile robots, which are capable of doing difficult jobs with relatively lower cost, gain a growing interest in the robotic field. In this paper, a model is used for a second order non-linear system to simulate a group of agents that interact via pair-wise attractive and repulsive potentials. a new potential field method is proposed using individually based agent perceptual model to successfully solve the local minimum problem, which is considered the main drawback in the artificial potential field based path-planning approach. The work in this paper is considered an extension of my earlier work to overcome local minima using internal agent states [1]. Allowing the potential field to be dynamic and manipulated by the model can solve the problem even easier. Simulation results demonstrate the ability of a single agent as well as group of agents to solve the problem and reach the goal position effectively.
\end{abstract}

\section{Key Words}

Robotics, Artificial potential field, path-planning.

\footnotetext{
${ }^{*}$ Egyptian Armed Forces
} 


\section{Introduction:}

The design and control of multi-agent robotic systems to accomplish specific missions has drawn considerable interest in recent years [2]. As teams of self-organizing agents that exchange information may have a greater functionality than the individual members, the operating principles of natural systems have proven to be a useful tool for the design and control of teams of robots that contain large number of individuals $[3,4]$. One of the main current trends is to employ teams of mobile robots in a range of applications in both civilian and military fields from space and sub-sea exploration to the deployment of teams of interacting artificial agents in disposal systems [5].

True artificial team design has been largely developed through two main approaches. The first is based on artificial physics [6] while the second is based on a set of practical, algorithmic approaches [7-10]. Both approaches have been applied to teams of autonomous agents and new assumptions about the architecture needed for intelligence have been investigated [11]. These approaches attempt to emulate natural, rather than artificial intelligence and are based on, or at least inspired by, biology. Natural examples of interacting individuals can be found in ants, bees, birds and schools of fish in the way that useful group properties can emerge through the interactions among the agents [12, 13].

Multi-agent robotic systems are often modelled as point mass agents in two-dimensional environments in which members may interact with one another through pair-wise attractiverepulsive interactions. Introducing agents with special tasks among groups, such as group virtual leaders [5,14-15], has also been introduced to provide provable group behaviour to ensure vehicles can avoid obstacles during navigation to a goal position. The actual realizations of self-propelled vehicles interacting according to virtual Morse potentials have been reported in the robotics literature $[5,16]$. These studies assume that the free parameters of the potential field are fixed a priori. In earlier work [1], the parameters were assumed to be internal states for each agent through which the agent can manipulate the potential field. The dynamics of these internal states were defined through set of first order differential equations that are applied on a collective basis. However effective, the aggregation behaviour has a main disadvantage as the entire team may be trapped in a dead end without having the chance to get help from one of the team members. This paper introduces the solution in such case by adopting an individual, rather than collective, behaviour of the team members along with increasing the perception of the agents about the environment and then allowing this perceptual model to manipulate the potential fields such that the problem can be solved easily and efficiently.

In this paper a model, which includes control forces that are induced by the gradient of the potential field, is used. The model consists of $N_{p}$ agents, it is considered here for the ${ }^{\text {th }}$ agent with mass $m_{i}$, position $\mathbf{r}_{i}$ and velocity $\mathbf{v}_{i}$. A dissipative friction force with coefficient $\beta_{i}$ is added to control the $i^{\text {th }}$ agent's speed. The global potential affecting the $i^{\text {th }}$ agent is characterised by attractive goal and repulsive obstacle potential fields of strength $C_{i g}$ and $C_{i o}$ with ranges $I_{i g}$ and $I_{i o}$ respectively and an agent interaction potential function $V_{\text {interaction }}\left(\mathbf{r}_{i}\right)$ that includes only the agent's repulsive potential field of strength $C_{r i}$ with range $I_{r i}$. This means of defining the interaction potential matches studies of pedestrian dynamics [17], which define each agent to have no attractive force to any of the other agents in the environment. The equations of motion of the $i^{\text {th }}$ agents are defined as: 


$$
\begin{aligned}
& v_{i}=\vec{r}_{i} \\
& m_{i} \vec{v}_{i}=-\beta v_{i}-\nabla_{i} V_{g l o b a l}\left(\boldsymbol{r}_{i}\right) \\
& V_{\text {global }}=V_{\text {repulsion/agents }}+V_{\text {repulsion/obstacles }}+V_{\text {attraction/goal }} \\
& V_{\text {global }}\left(\mathbf{r}_{i}\right)=\sum_{j \neq i}^{N_{p}} C_{r_{j}} e^{-\left|\mathbf{r}_{i}-\mathbf{r}_{j}\right| / l_{r_{j}}}+\sum_{z=1}^{N_{o}} C_{i o_{z}} e^{-\left|\mathbf{r}_{i}-\mathbf{r}_{o_{z}}\right| / l_{i o z}}-C_{i g} e^{-\mathbf{r}_{i}-\mathbf{r}_{g} \mid / l_{i g}}
\end{aligned}
$$

where $V_{\text {globa }}\left(\mathbf{r}_{i}\right)$ is the global potential corresponding to the th agent, $V_{\text {repulsion/agents }}$ is the repulsion potential amongst the agents, $V_{\text {repulsion/obstacles }}$ is the repulsion potential between each agent and the obstacles, $V_{\text {attraction/goal }}$ is the attraction potential to the goal. Using an Adams-Bashforth numerical integration method a simulation code has been developed to predict the collective behaviour of the agents, which were given random initial positions and velocities.

\section{Problem Definition:}

The problem of local minima (trapped situation), shown in Fig. 1, was discussed by many researchers [18]. The reactive problem for an agent, or team of agents, attracted to a goal point at position $G$ can be defined such that an artificial potential field at $G$ induces motion towards the goal. When the agent, or team of agents, moves towards the goal the velocity of each individual agent rises, and the agents translate to the goal along the gradient of the potential field. However, in order prevent collision with a static obstacle, an additional repulsive potential field is required.

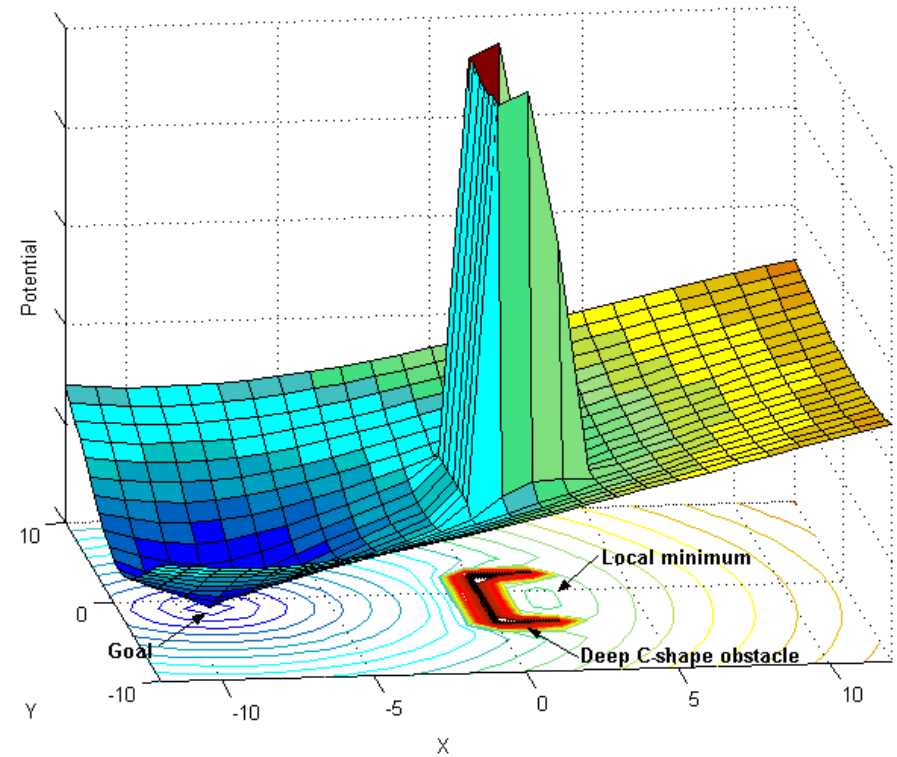

Fig.1. Classical reactive problem 
These two potential fields are then superimposed to form a global potential field that describes the workspace of the problem. In general however, a local minimum may form due to the superposition of the goal potential and that of the obstacles, resulting in the agent, or team of agents, becoming trapped in a state other than the goal $G$.

Considering this problem, the entire team, or part of the team will be trapped at the obstacle since the agents trapped inside the obstacle will experience two virtual forces; the first force is the attraction to the goal while the other will be the repulsion from the obstacle. Moreover in most cases there will be no opportunity for the team members to escape from the local minimum due to the pair-wise interaction potential - particularly when the goal potential is of large amplitude.

\section{Squeeze Effect:}

Escape from complex workspaces can be seen in many natural systems in which the system consists of a number of agents enclosed in a trap. In order to illustrate the use of the agent interaction parameters, a problem in which the phenomenon of translation of agents from a region of high potential to low potential can be investigated (e.g. system of gas molecules whose individuals are enclosed in a single-exit container while they experience a change in their state such as a rise in temperature).

Let $(A, B)$ be two adjacent regions, as shown in Fig. 2, such that both have the same global potential. Using dynamic interaction parameters for a group of agents enclosed in a trap, the change of the interaction potential range of the system individuals, $I_{r i}$, simply changes the trap region from a local minimum into a region of maximum potential from which all the agents are emitted as if squeezed out, as shown in Fig. 2.

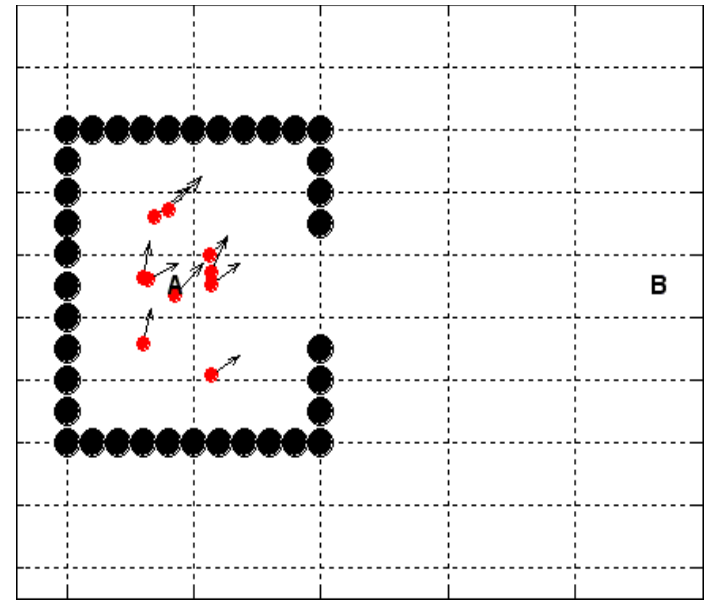

(a)

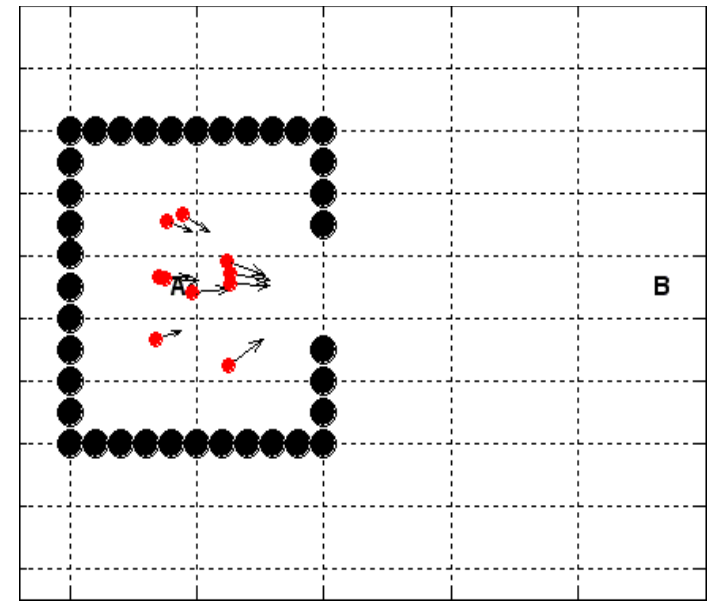

(b) 


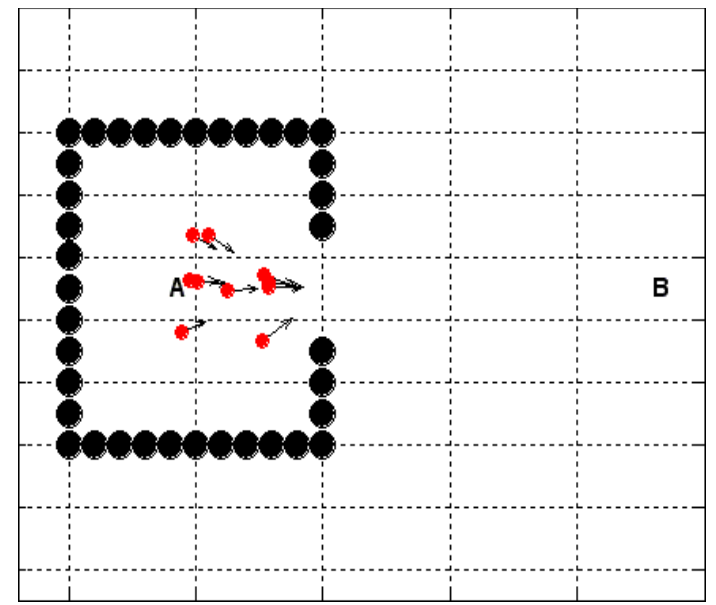

(c)

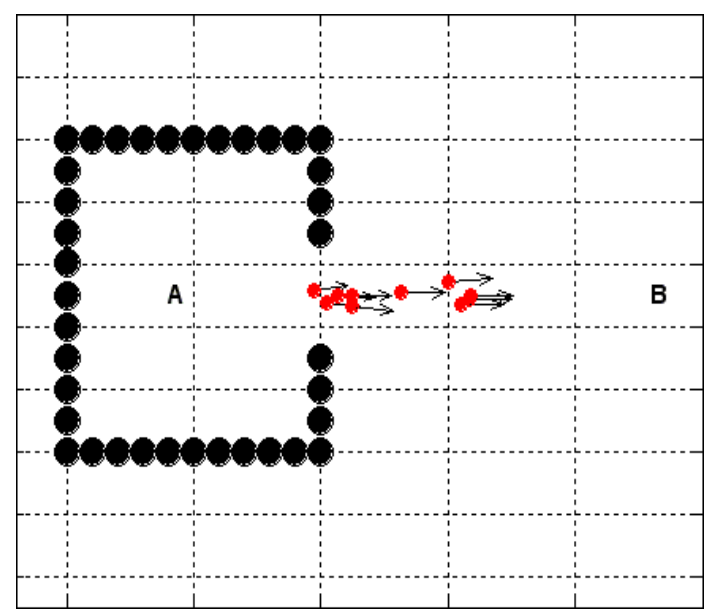

(e)

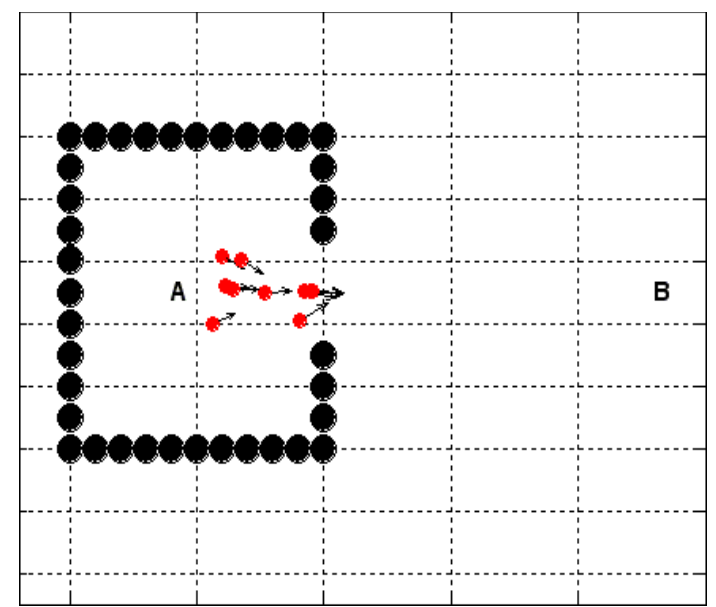

(d)

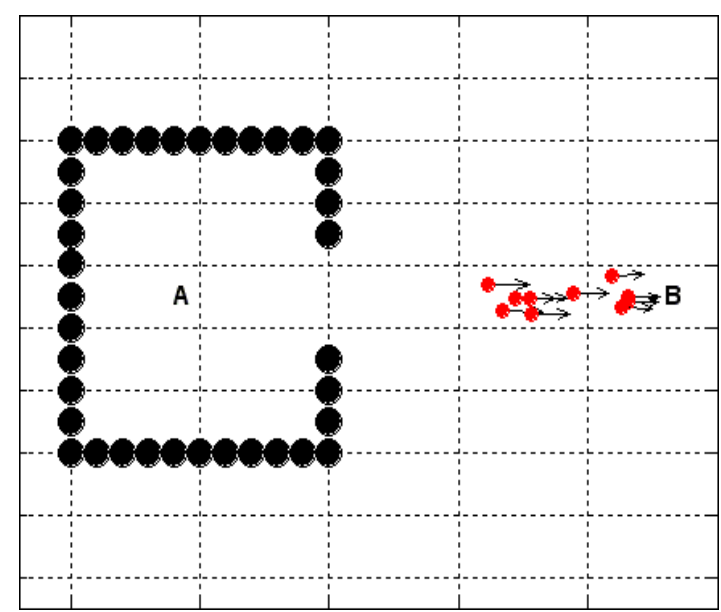

(f)

Fig. 2. Squeeze effect

The use of agent perceptual model will now be considered as a means of allowing agents to manipulate the potential field in which they are maneuvering in order to escape from local minima.

\section{Agent Perceptual Model:}

The previous section has demonstrated that a change in the interaction potential parameters of the agents can lead to escape from a trap (manipulating a local minimum into a local maximum). This concept will now be used for a single agent maneuvering towards a goal in a potential field, which contains a local minimum.

For a fixed obstacle, the repulsion potential range affecting the $i^{\text {th }}$ agent $\left(l_{i 0}\right)$ can be represented as a function of an obstacle constant $\left(I_{0}\right)$, which characterizes the physical nature of the obstacle, and the particle repulsion potential range $\left(I_{r i}\right)$ which characterizes the agent interaction potential range. The attraction potential range of the goal affecting the th agent $\left(l_{i g}\right)$ can also be represented as a function of a goal constant $\left(I_{g}\right)$, which characterizes the physical nature of the goal, such that: 


$$
\begin{aligned}
& C_{i o}=C_{o} \\
& l_{i o}=l_{o}+l_{r i} \\
& C_{i g}=C_{g} \\
& l_{i g}=l_{g}
\end{aligned}
$$

When an agent approaches an obstacle it suffers an elastic collision which pushes the agent away from the goal. The goal then attracts the agent back and the agent will never attempt to maneuver around the obstacle simply because it never knows it's trapped.

The agent's perception function $Q_{i}$ is now defined, inspired from the learning by reward or punishment [19-20], and applied to the agent and its ability to perceive the environment in which it exists. The function is defined as the change of the modulus of the agent's velocity measured in some interval of time interval. If the agent is repulsed from an obstacle $Q_{i}$ will have a negative value which is punishment, because the agent is moving away from the goal. If the agent is moving towards the goal $Q_{i}$ will have a positive value which is the reward as the agent senses it is moving to the goal.

When an agent enters the zone of the local minimum, as demonstrated in Fig. 1, it suffers an elastic collision that pushes the agent away from the goal. The goal attracts it back, while the repulsion effect decays as the agent moves away from the obstacle. These two forces will lead the agent to reduce its velocity until it stops. The agent will never attempt to manoeuvre around the barrier simply because it never knows it is trapped. The function $\left(Q_{i}\right)$ is now used successfully to make the agent realise that it is trapped and that it should change its interaction characteristics. Specifically, increasing its repulsion potential parameters to change the obstacle zone from a region of local minimum to a region of maximum potential, as discussed in Section 3. In this way the agent will be emitted from the obstacle zone and a minimum gradient potential path is defined during relaxation of the potential as soon as the problem is solved (i.e. $Q_{i}$ has a positive value again). This gradient path will lead the agent directly to the goal to avoid becoming trapped again.

This new algorithm allows the free parameters of the potential field to be dynamic and couples the agent's interaction parameters with its perception about its progress through the workspace. If the agent is progressing towards the goal, or its position $\mathbf{r}_{i}$ relative to the goal position $\mathbf{r}_{g}$ is less than some small limit $\varepsilon$, then the parameters are fixed. The agent interaction parameters (potential field free parameters) will now be defined through a set of differential equations, which will allow the agents, on an individual basis, to manipulate the potential field in which they are maneuvering. The following set of differential equations are used to express the interaction parameters of the agents: 


$$
\begin{aligned}
& \dot{C}_{r i}=\left\{\begin{array}{cc}
A_{r} e^{-\lambda_{q} Q_{i}}-\lambda_{r} C_{r i} & \text { if } Q_{i}<0 \\
0 & \text { if }\left(Q_{i} \geq 0\right) \text { or }\left|\mathbf{r}_{i}-\mathbf{r}_{g}\right| \leq \varepsilon
\end{array}\right. \\
& \dot{l}_{r i}= \begin{cases}B_{r} e^{-\lambda_{q} Q_{i}}-\lambda_{r} l_{r i} & \text { if } Q_{i}<0 \\
0 & \text { if }\left(Q_{i} \geq 0\right) \text { or }\left|\mathbf{r}_{i}-\mathbf{r}_{g}\right| \leq \varepsilon\end{cases} \\
& \dot{\beta}_{i}= \begin{cases}A_{\beta} e^{-\alpha_{q} Q_{i}}-\lambda_{\beta} \beta_{i} & \text { if } Q_{i}<0 \\
0 & \text { if } Q_{i} \geq 0\end{cases}
\end{aligned}
$$

Eq. (9-10) express the repulsion amplitude and range of the $i^{\text {th }}$ agent, according to the use of the function $Q_{i}$. For $\left(Q_{i}<0\right)$ it can be seen that the agent will experience repulsion which leads to motion away from obstacles. Moreover, Eq. (11) ensures a smooth manoeuvre around the obstacle by slowing the agent. The damping terms in Eq. (9-11) ensure smooth and quick relaxation of the global potential field as soon as the problem is solved.

The benefit of the function $Q_{i}$ is that when the agent is repelled $\left(Q_{i}<0\right)$, lio takes a high value, which turns the workspace in the neighbourhood of the obstacles into a zone of maximum potential. This then leads to escape from the local minimum (Section 3), with the potential field relaxing after escape due to the damping terms in the differential equations for the interaction parameters. The relaxation effect then defines a gradient path, which the agent can follow to go directly to the goal. The control parameters (coefficients) $A_{r}, B_{r}, A_{\beta}, \lambda_{r}, \lambda_{q}, \lambda_{\beta}$ and $\alpha_{q}$ are employed to scale the dynamics of the interaction parameters. The model analysis for a single agent is now considered.

\section{Simulation Results:}

The simulation shows that using the dynamic perceptual model, Eqs. (5-11), the potential field around the obstacle is converted from a local minimum into a local maximum. This will ensure the agent is pushed from the obstacles and will maneuver around them. After the agent is squeezed away it will follow the gradient of the potential field around the obstacles and approach the goal $G$.

First, the case of an agent with fixed interaction parameters is considered. Here the free parameters describing the potential field, and so the potential field itself, are constant. The contour map shown in Fig. 3 is static and so the agent becomes trapped in the local minimum of the potential field. This is typical of conventional implementations of the artificial potential field method to path planning problems.

For dynamical interaction parameters the contour map in Fig. 4 shows that the agent enters the local minimum, and when repelled $\left(Q_{<} 0\right)$ the repulsion potential of the agent increases in 
a way that converts the obstacle to be a zone of maximum potential to the agent. As the agent escapes from the local minimum the potential field relaxes due to the damping terms in Eqs. (9-11). The goal potential field then drags the agent away from the obstacle zone and defines a gradient path that the agent follows directly to the goal. The comparison between the contour maps in Fig. 3 and Fig. 4 shows clearly the effect of using the function $Q_{i}$ and agent's perceptual model to solve the reactive problem effectively.

Finally, the simulation results, shown in Fig. 5, demonstrate the behaviour of a group of agents that adopt the individual based algorithm to solve the local minimum problem for unit control parameters for the same goal and obstacles parameters used for the problem definition. The simulation results, demonstrated in Fig. 5, show the efficiency of the model to the solve the problem for a group of agents.

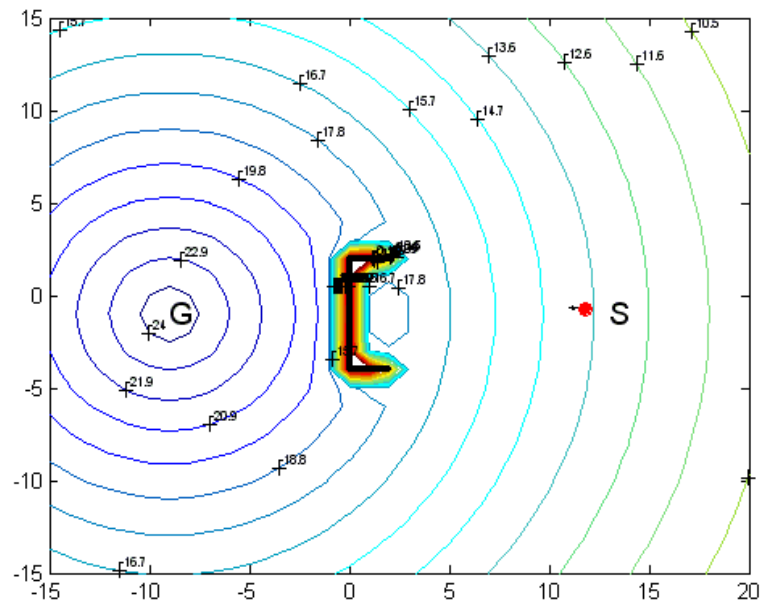

(a)

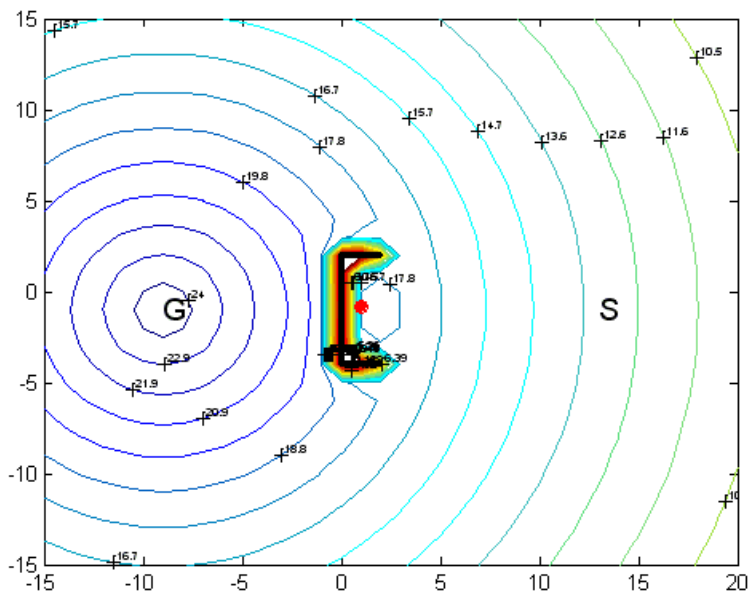

(b)

Fig. 3. Behaviour of a conventional agent with fixed interaction parameters

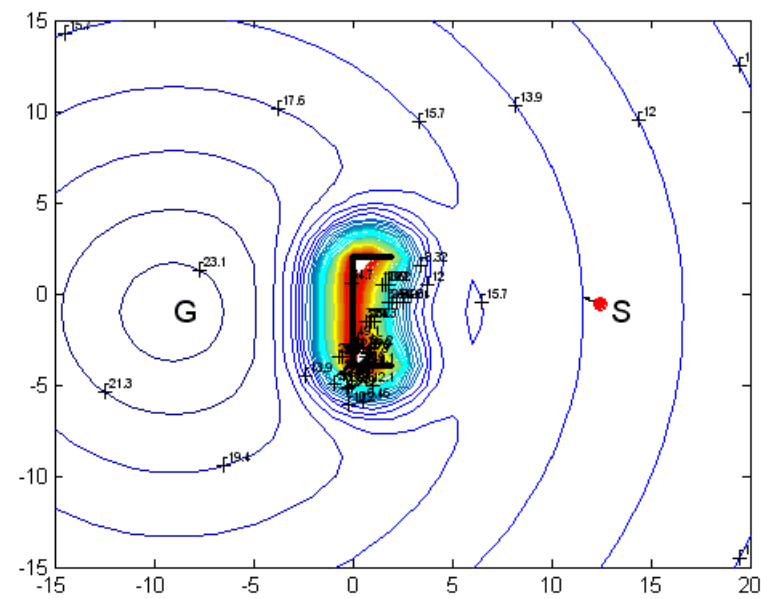

(a)

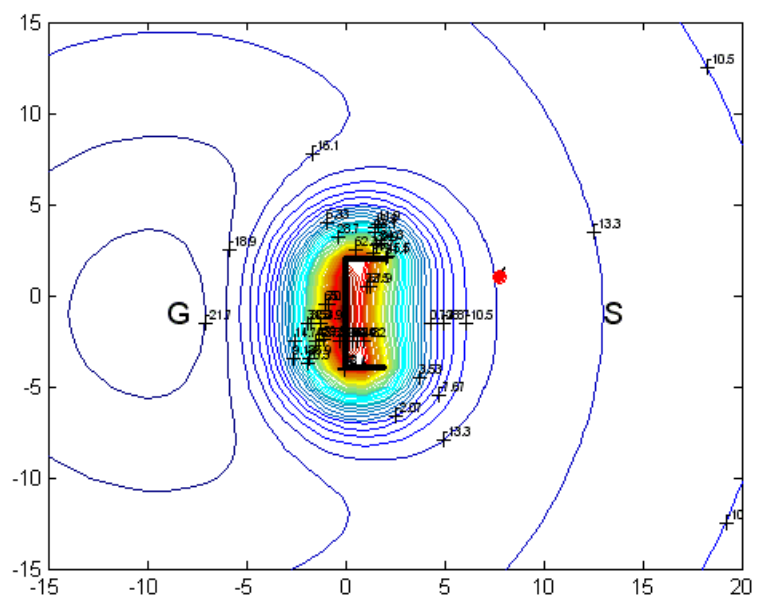

(b) 


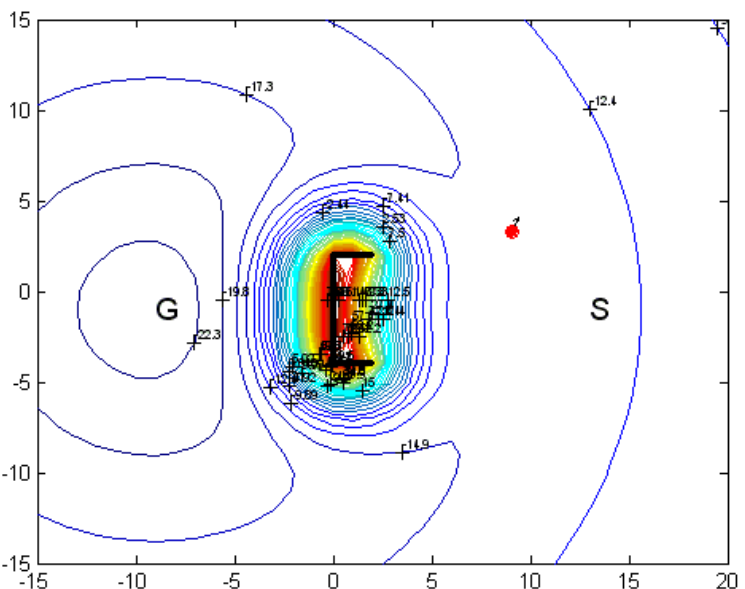

(c)

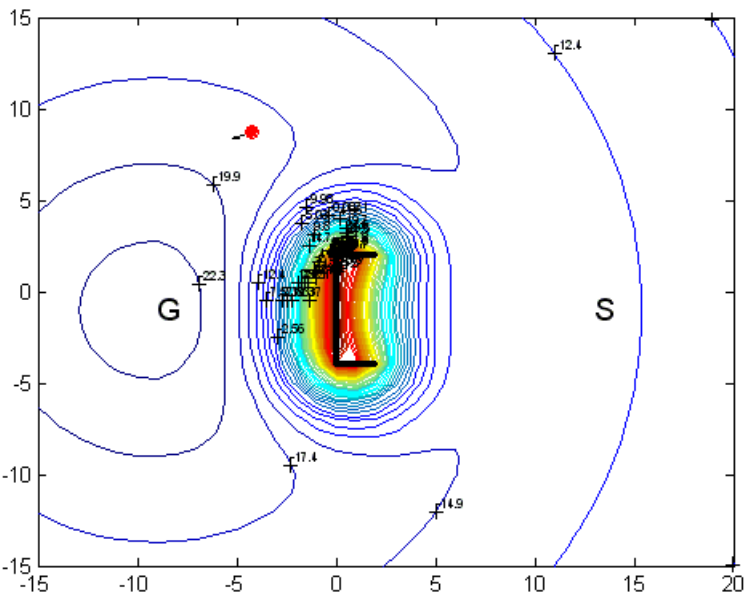

(e)

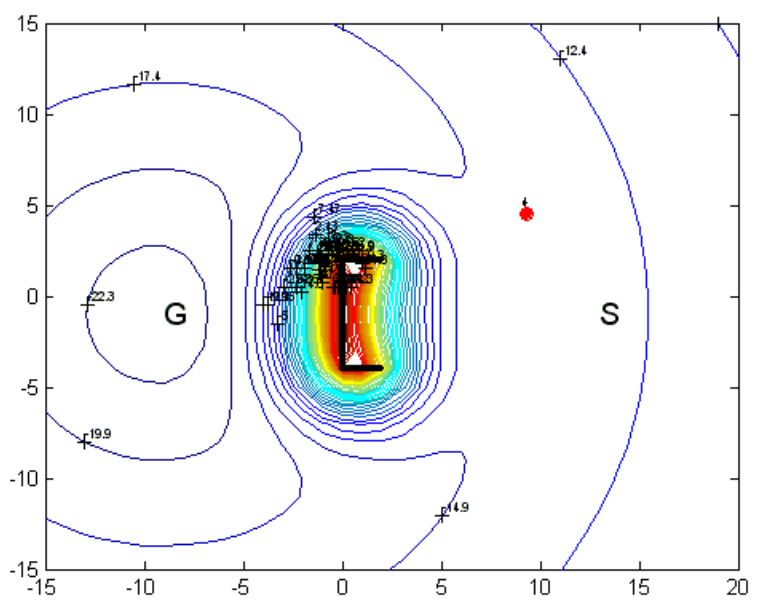

(d)

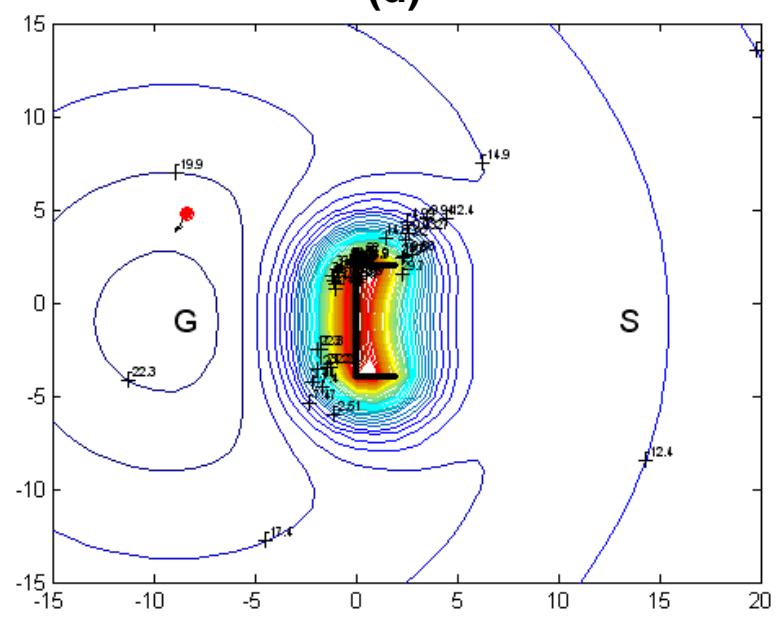

(f)

Fig. 4. Behaviour of an agent using the perceptual model

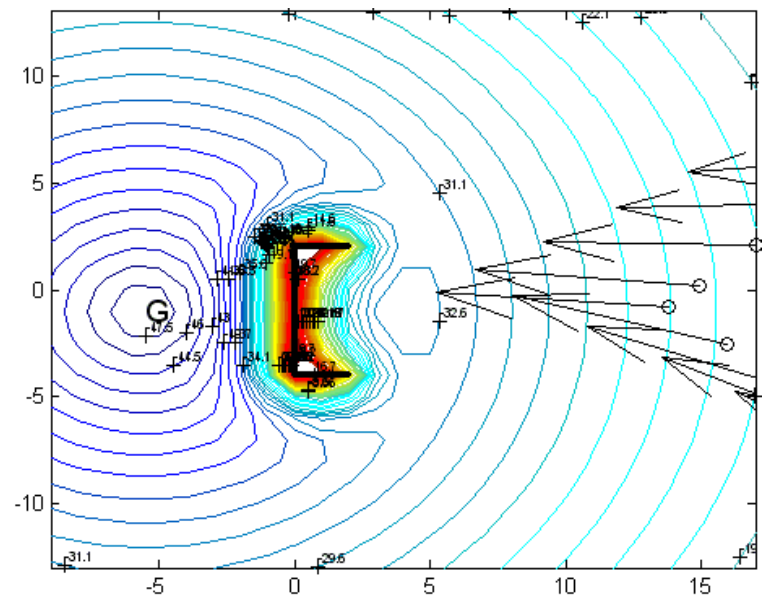

(a)

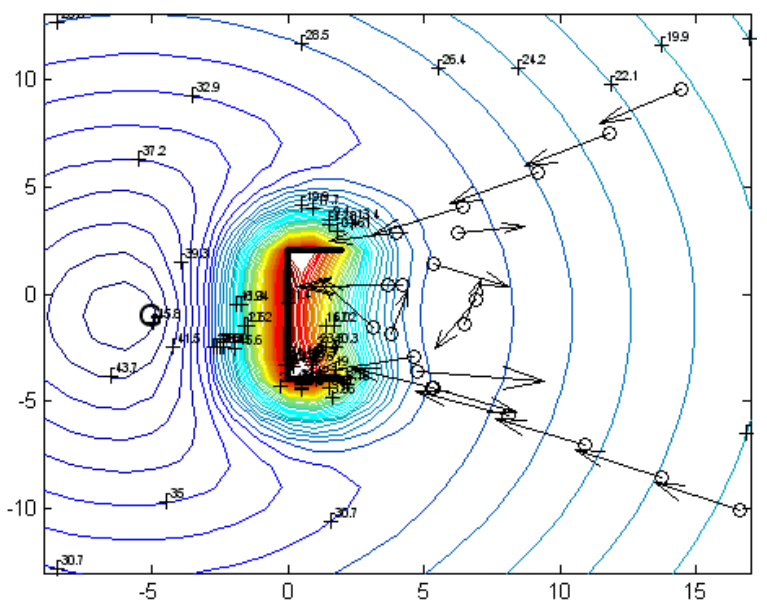

(b) 


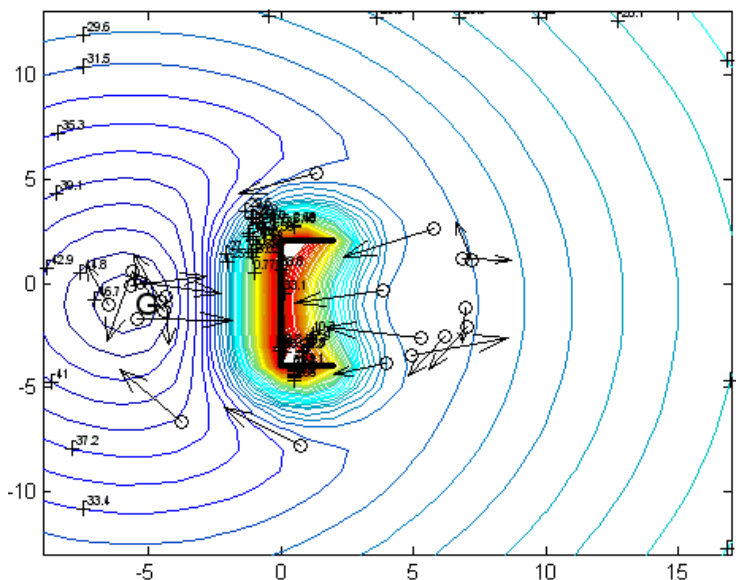

(c)

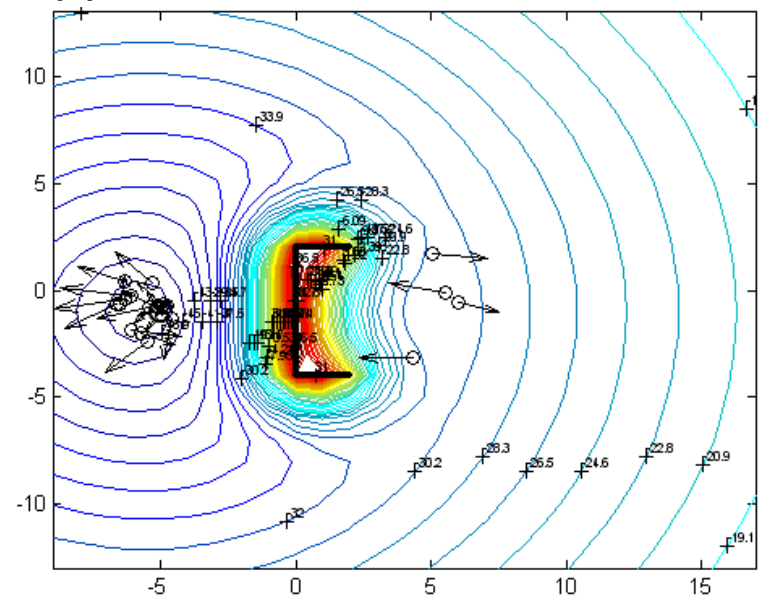

(e)

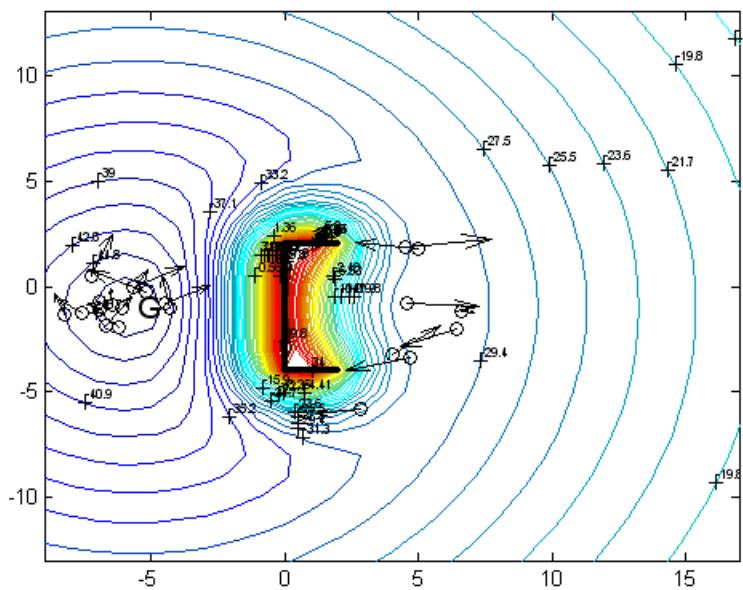

(d)

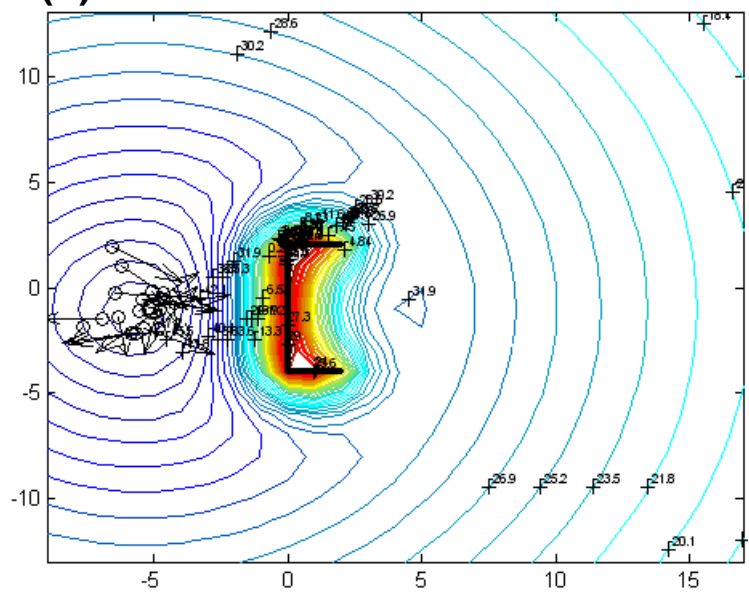

(f)

Fig. 5. Behaviour of a group of agents using the perceptual model

\section{Conclusions:}

This paper introduces and extension of earlier work which is based on solving the local minimum problem using the aggregation concept [1]. However effective, the aggregation behaviour has a main disadvantage when the entire team may be trapped in a dead end without having the chance to get help from one of the team members. This paper introduces the solution in such case by adopting an individual, rather than collective, behaviour of the team members that are cabala of solving the local minimum problem. The new method uses the concept of increasing the perception of the agents about the environment and then allowing this perceptual model to manipulate the potential fields such that the problem can be solved easily and efficiently. The method allows a team of agents to escape from and maneuver around a local minimum in the potential field to reach a goal. Rather than moving in a static potential field, the agents, on an individual basis, are able to manipulate the potential according to their estimation of whether they are moving towards or away from the goal. Using the agent's perception function $Q_{i}$ along with the use of the squeeze effect, simulation results show that a team of agents can effectively escape from a local minimum. 


\section{References:}

[1] M. Mabrouk, "Overcoming Local Minima in Artificial Potential Field Path-Planning Using Perceptual Agent States", PhD thesis, University of Strathclyde, 2009.

[2] E W Justh, P S Krishnaprasad, "Steering Laws and Continuum Models for Planar Formations", Proceedings of the 42nd IEEE International Conference on Decision and Control 3609-3614,2003

[3] E. Bonabeau, M. Dorigo, and G. Theraulaz, "Swarm Intelligence: From Natural to Artificial Systems", Oxford University Press, New York, 1999.

[4] V. Gazi and K. Passino, "Stability Analysis of Swarms", in IEEE Trans. Autom. Contr. Vol. 48, pp. 692-697, 2003.

[5] N. E. Leonard and E. Fiorelli, "Virtual Leaders, Artificial Potentials, and Coordinated Control of Groups", in Proc. Conf. Decision Contr., Orlando, FL, pp. 2968-2973, 2001.

[6] Spears W., and Gordon D., "Using Artificial Physics to Control Agent", Proceedings of IEEE International Conference on Information, Intelligence, and Systems (ICIIS'99), November, 1999.

[7] Kelly I. and Keating D, "Faster Learning of Control Parameters Through Sharing Experiences of Autonomous Mobile Robots", International Journal of Systems Science. 29(7), 783-793, 1998.

[8] Mataric M., "Designing and Understanding Adaptive Group Behaviour", Adaptive Behavior, 4 (1): 51-80, 1995.

[9] Mataric M., "Issues and Approaches in the Design of Collective Autonomous Agents", Robotics and Autonomous Systems, 16 (2-4): 321-331, 1995.

[10] Mataric M., "Behaviour-Based Control: Examples From Navigation, Learning, and Group Behaviour", Journal of Experimental and Theoretical Artificial Intelligence, 9 (2-3): 323-336, 1997.

[11] Balkenius, C. "Natural Intelligence for Autonomous Agents", (extended version), Lund University Cognitive Studies, 29,1994.

[12] S. Camazine, J. L. Deneubourg, N. R. Franks, J. Sneyd, G. Theraulaz, and E. Bonabeau, "Self Organization in Biological Systems", Princeton University Press, Princeton, NJ, 2003.

[13] J K Parrish, L Edelstein-Keshet, "Complexity, Pattern, and Evolutionary Tradeoffs in Animal Aggregation", Science 284:99-101, 1999.

[14] D. Dimarogonas, M. Egerstedt and K. Kyriakopoulos "A leader-based containment control strategy for multiple unicycles" In Proc. of the IEEE Conference on Decision and Control, 5968-5973, 2006.

[15] M. Mabrouk, "Using the Swarm Leader Concept for Multi-agent Obstacle Avoidance", accepted in the Al-Azhar Engineering Eleventh International Conference, Cairo, Egypt, 2010.

[16] B. Q. Nguyen, Y. L. Chuang, D. Tung, C. Hsieh, Z. Jin, L. Shi, D. Marthaler, A. L. Bertozzi, and R. M. Murray, "Virtual Attractive Repulsive Potentials for Cooperative Control of Second Order Dynamic Vehicles on the Caltech MVWT", in Proc. American Contr. Conf., Portland, OR, pp. 1084-1089, 2005.

[17] D. Helbing and P. Molnar, "Social force model for pedestrian dynamics", Physical Review E 51, (5) 4282-4286, 1995.

[18] H. Moradi, H. Suyoen, L. Sueng and L. Dohyung, "Local minima avoidance", 
Intelligent Systems Research Center technical notes, TR-MP-20050913-0.2, 2005.

[19] Mackintosh, N. J., (1983), "Conditioning and Associative Learning”, Oxford: Oxford University Press.

[20] Lieberman, D. A., (1990), "Learning: Behaviour and Cognition", Belmont, CA: Wadsworth Publishing Company.

\section{Nomenclature}

$\begin{array}{ll}C_{a i} & \text { Amplitude of attraction potential of the } \mathrm{i}^{\text {th }} \text { ag } \\ C_{i g} & \text { Amplitude of goal total potential affecting the } \\ C_{i o} & \text { Amplitude of obstacle potential affecting } \mathrm{i}^{\text {th }} \\ C_{r i} & \text { Amplitude of repulsive potential of the } \mathrm{i}^{\mathrm{h}} \text { ag } \\ L_{i a} & \text { Range of attractive potential for the } \mathrm{i}^{\text {th }} \text { agen } \\ L_{i g} & \text { Range of goal potential affecting the } \mathrm{i}^{\text {th }} \text { agent } \\ l_{i o} & \text { Range of obstacle potential affecting the } \mathrm{i}^{\text {th }} \\ I_{g} & \text { Range of goal potential which characterizes } \\ I_{o} & \text { Range of obstacle potential which characte } \\ & \text { obstacle } \\ I_{r i} & \text { Range of repulsive potential of the } \mathrm{i}^{\text {th }} \text { agent } \\ m_{i} & \text { Mass of the } \mathrm{i}^{\text {th }} \text { agent } \\ N_{p} & \text { Number of agents } \\ \mathbf{r}_{i g} & \text { Goal }-\mathrm{i}^{\text {th }} \text { agent position vector } \\ \mathbf{r}_{i o} & \text { Obstacle }-\mathrm{i}^{\text {th }} \text { agent position vector } \\ V_{g l o b a l}\left(\mathbf{r}_{i}\right) & \text { Generalized Morse potential } \\ V_{g o a}\left(\mathbf{r}_{g}\right) & \text { Goal potential field } \\ V_{\text {interaction }}\left(\mathbf{r}_{i}\right) & \mathrm{i}^{\text {th }} \text { agent interaction potential } \\ \mathbf{v}_{i} & \mathrm{i}^{\text {th }} \text { agent velocity vector } \\ Q_{i} & \text { Agent's perception function } \\ \beta & \text { Friction self-decelerating force coefficient }\end{array}$

